HEALTH SCIENCES

\title{
Studies on the characterization and polymorphic stability of Fosamprenavir
}

\author{
CLEYDSON F. CORDEIRO, INGRID BETTIO \& MARCELLO G. TREVISAN
}

\begin{abstract}
Fosamprenavir calcium is an amprenavir prodrug of the protease inhibitors class used in the treatment of patients with acquired immunodeficiency syndrome (AIDS). Different solid forms of this drug are described in patents, in this sense studies on the physico-chemical characterization and stability are relevant for the selection of a solid form with adequate features for pharmaceutical purposes. In the present work form I (commercial) and amorphous of fosamprenavir calcium were characterized by the techniques of Differential Scanning Calorimetry (DSC), Thermogravimetry (TGA), Powder X-ray Diffraction (PXRD), Fourier-Transform Infrared Spectroscopy (FTIR) and Scanning Electron Microscopy (SEM). Furthermore, the chemical and polymorphic stability of the commercial form were evaluated by DSC, PXRD, FTIR and High-Performance Liquid Chromatography (HPLC). In the studies of characterization, thermal analyses allied to spectroscopic methods (PXRD and FTIR) demonstrated that the presence of water in the crystalline structure of Form I is fundamental for maintaining its crystallinity. In studies of accelerated stability the techniques of DSC, PXRD and FTIR showed that Form I does not suffer phase change when submitted to controlled conditions of temperature and humidity. Moreover, HPLC and FTIR proved the chemical stability of this solid form of fosamprenavir, thus demonstrating its suitability for pharmaceutical purposes.
\end{abstract}

Key words: Fosamprenavir, characterization, stability, Polymorphism.

\section{INTRODUCTION}

The world trade of pharmaceutical products has presented a significant growth in the last decades (WHO 2002). This has attracted the attention of pharmaceutical industries and regulatory agencies regarding technical and scientific aspects related to the security and efficiency of these products. In this context, the development of studies on the characterization and stability of drugs play a relevant role to guarantee the quality of offered products (Singh et al. 2012).

The characterization of drugs represents an important step for the development of new products (Shete et al. 2010). Most of the active pharmaceutical supplies may exist under different solid forms, such as crystalline or amorphous material, which may present distinct physicochemical and biopharmaceutical properties. In this sense, selecting the solid form of a drug with suitable properties is fundamental for the therapeutic success of a formulation (Shete et al. 2012, Santos et al. 2016). Different properties of the drug may be evaluated on essays of characterization, such as the polymorphic form, crystallinity, morphology and thermal stability. Several analytical techniques have been used for this purpose, especially thermal analyses and spectroscopic methods (Daniel et al. 2013, Chadha \& Bhandari 2014). 
Studies of stability represent a crucial point for the development of high-quality pharmaceutical products (Correa et al. 2016), that is why regulatory agencies have provided guides and resolutions recommending assays under the influence of different environmental factors, such as temperature and humidity (Anvisa 2012, ICH 2003). Evaluating the influence of such parameters is relevant since their chemical stability may be altered, thus forming toxic products of degradation or even alterations of the crystalline phase (Júlio et al. 2015).

The fosamprenavir calcium (Figure 1) is a prodrug of the class of protease inhibitors, which is hydrolyzed to its active form, the amprenavir. It is used in the antiretroviral therapy to combat the AIDS virus, with pharmacokinetic advantages

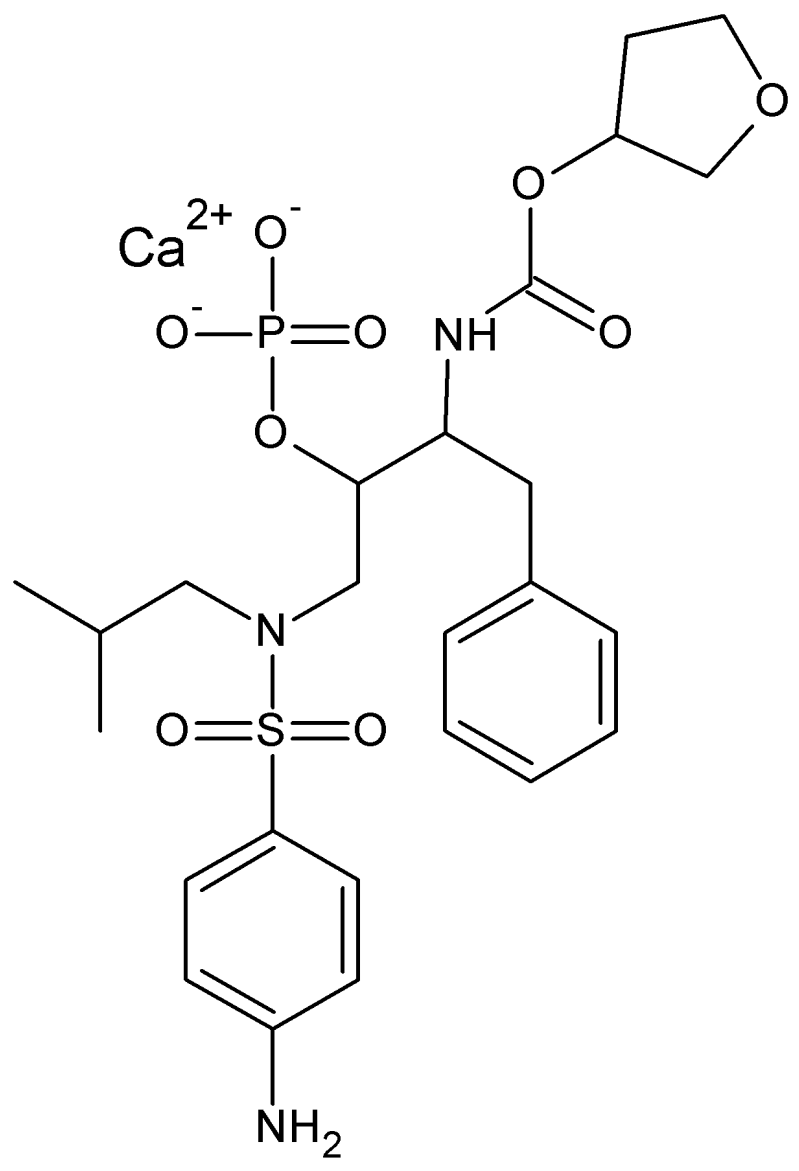

Figure 1. Chemical structure of fosamprenavir calcium. in relation to the original drug, which allows a better posology and less pain, thus favoring the acceptance by patients (Rossi et al. 2011). Fosamprenavir is called type II according to the system of biopharmaceutical classification, with low solubility and high permeability. Such characteristics are critical factors for polymorphic drugs (Rossi et al. 2011).

Different crystalline forms of fosamprenavir calcium are described in patents: Form I (Hydrate) (Armitage et al. 2003), Form II (Bhoge et al. 2012), Form III (Leksic et al. 2011), Form IV [solvate], Form P (Leksic et al. 2011), Form A (Jetti et al. 2013) and Form H1 (Reddy et al. 2015). Furthermore, methods are reported to obtain amorphous forms of this drug (Leksic et al. 2011), although any work was found in the literature reporting the characterization of such forms or the stability of crystalline phases. In this sense, it is relevant to conduct such assays since possible phase transitions or degradations of the pharmaceutical ingredient may hamper the quality, security and efficiency of commercial preparations of this drug. Therefore the present work aimed the characterization of fosamprenavir calcium Form I and amorphous and its chemical/polymorphic stability at controlled conditions of temperature $\left(40^{\circ} \mathrm{C}\right)$ and humidity $(75 \% \mathrm{RH})$. Samples were characterized by the techniques of TGA, DSC, FTIR, PXRD and SEM. The polymorphic stability was evaluated by Thermal Analysis (DSC) applying FTIR and PXRD as complementary techniques. The chemical stability was determined by HPLC and FTIR.

\section{MATERIALS AND METHODS}

The fosamprenavir calcium was obtained from the reference medicine Telzir ${ }^{\circledR}$, which is marketed as coated tablets. The commercial lots 7262 and 3R9G produced by the laboratory 
Glaxo Operations UK Limited were used as bulk for purification. The medicine was provided by the Ministry of Health (Brazil)/ National Program of Sexually transmitted diseases and AIDS. After extraction, samples of fosamprenavir were characterized by the techniques FTIR, liquid chromatography coupled to mass spectrometry (LC-MS/MS) and nuclear magnetic resonance of ${ }^{13} \mathrm{C}$ and ${ }^{1} \mathrm{H}$. The chromatographic purity of the extracted drug was determined by HPLC through a method developed and validated in our laboratory, revealing the value $99.95 \%$.

The reagents methanol HPLC grade (Tédia, Fairfield, USA), acetonitrile HPLC grade (SigmaAldrich, St. Louis, USA), ammonium acetate analytical grade (ISOFAR, Duque de Caxias, Brazil), acetic acid analytical grade (SigmaAldrich, St. Louis, USA), triethylamine analytical grade (Merck, Darmstadt, Germany), ethanol analytical grade (Vetec, Duque de Caxias, Brazil) and purified water obtained by Milli-Q Academic (Millipore, São Paulo, Brazil) were used in the analytical methods.

\section{Preparations of solid forms of fosamprenavir calcium}

Form l: solid form of fosamprenavir calcium obtained from Telzir ${ }^{\circledR}$. The extraction was made based on the method described in the patent (Bhoge et al. 2012) for the crystallization of this form. Initially the mean weight of 20 Telzir ${ }^{\circledR}$ tablets was determined. The coating of tables was removed and they were ground until obtaining fine and homogeneous powder. A sample of $5 \mathrm{~g}$ of the medicine was weighed and transferred to a round bottom flask and 75 $\mathrm{mL}$ of a solution of methanol and ethanol 95:5 (/v) were added. The sample was placed in an ultrasonic bath during $20 \mathrm{~min}$ and posteriorly heated in bath at $70{ }^{\circ} \mathrm{C}$ until obtaining a clear supernatant. Insoluble products were removed by filtration in filter paper and washed with
$25 \mathrm{~mL}$ of the solution ethanol-methanol. The filtrate was heated again at $70{ }^{\circ} \mathrm{C}$ until obtaining a clear solution and $15 \mathrm{~mL}$ of purified water was added. The sample was slowly cooled until $25^{\circ} \mathrm{C}$ and kept under agitation during $3 \mathrm{~h}$. The resulting product was filtered, washed with 20 $\mathrm{mL}$ of solution of ethanol and purified water 50:50 ( $/ \mathrm{v}$ ) and posteriorly dried in stove at 40 ${ }^{\circ} \mathrm{C}$ until constant weight. Form I of fosamprenavir calcium was characterized by the techniques PXRD, TGA, DSC, FTIR and SEM.

Amorphous: Approximately $5 \mathrm{~g}$ of Form I of fosamprenavir calcium were conditioned in Petri dishes and placed in stove at $100{ }^{\circ} \mathrm{C}$ at vacuum during $5 \mathrm{~h}$. The amorphous form was characterized by the techniques PXRD, TGA, DSC and FTIR.

\section{Study of chemical and polymorphic stability}

Studies of stability were conducted using Form I of fosamprenavir calcium, the commercial crystalline phase of the medicine. Samples were analyzed by the techniques DSC, PXRD, FTIR and HPLC, which were incubated in stability chamber at $40^{\circ} \mathrm{C} \pm 2{ }^{\circ} \mathrm{C}$ and relative humidity of $75 \%$ in order to evaluate the influence of such parameters over the chemical and polymorphic stability of the drug. Analyses were conducted after three and six months of incubation applying the same techniques.

\section{Thermal Analysis}

DSC analysis were conducted in a calorimetric cell DSC model Q20 (TA Instruments) in the range of 30 to $350{ }^{\circ} \mathrm{C}$. TG analysis were conducted in a thermo-balance TGA/DTA 7300 (SII Nano Technology, Japan) in an interval from 30 to $500{ }^{\circ} \mathrm{C}$. Both analyses were conducted using approximately $3 \mathrm{mg}$ of sample and opened aluminum crucible with heating rate of 10 으. $\mathrm{min}^{-1}$ at a flow of $50 \mathrm{~mL}$. $\mathrm{min}^{-1}$ of nitrogen (purity 
over $99.995 \%)$. Both tequipments were calibrated using a standard metallic indium.

\section{Powder X-ray diffraction analysis}

Diffractograms were obtained by PXRD with angular scanning of $2 \theta$ between 3 and $40^{\circ}$ using $40 \mathrm{KV}$ and $30 \mathrm{~mA}$. The diffractometer was operated in the mode Step Scan with step of 0.02 - and integration time of 0.5 second per step. Copper was applied as source of X-rays (mean Ka: 1.54178) and nickel as $\beta$ filter. Analyses were conducted in an X-ray diffractometer Ultima IV (Rigaku diffractometer, Japan). Coating was removed from Telzir ${ }^{\circledR}$ tablets before PXRD analysis.

\section{Fourier transform infrared spectroscopy}

Absorption bands characteristic of the chemical structure of fosamprenavir calcium were obtained by FTIR at room environment from 4000 a $400 \mathrm{~cm}^{-1}$ using 32 scans and resolution of $4 \mathrm{~cm}^{-1}$. Analyses were made in an Infrared spectrometer Nicolet iS50 FTIR (Thermo Scientic, USA) coupled with an ATR accessory (Pike Technologies, USA).

\section{Scanning electron microscopy}

Electromicrographs of Form I were obtained at vacuum by scanning electron microscopy with acceleration voltage between $3 \mathrm{kV}$ and $6 \mathrm{kV}$. Samples were covered with gold film. Images were obtained with magnifications of $4000 x$, $8000 x$ and $13000 x$. Analyses were conducted in a scanning electron microscope model JSM6340F Field Emission, JEOL, Japan.

\section{High-performance liquid chromatography}

A method indicating stability through reverse phase HPLC was developed to determine the chromatographic purity of the medicine obtained from Telzir ${ }^{\circledR}$. This method was also applied to the stability study for drug dosing and quantification of the possible degradation products.

Analyses were conducted in UHPLC Ultimate 3000 LC system (Thermo Scientific, California, USA) equipped with detector DAD-3000RS and software Chromeleon 6.8. The method uses as mobile phase a mixture of ammonium acetate $20 \mathrm{mM}$ containing $0.1 \%$ of trimethylamine $(\mathrm{pH}$ 6.0 adjusted with acetic acid) and acetonitrile in the proportion 68:32 (v/v). After the preparation the mobile phase was filtered in membrane $0.22 \mu \mathrm{m}$ and degassed. A flow of $1.1 \mathrm{~mL} \cdot \mathrm{min}^{-1}$ and volume of injection of $20 \mu \mathrm{L}$ was applied. The chromatographic separation was conducted using a column Acclaim ${ }^{\circledR} 120$ C-8, 250 mm length, $4.6 \mathrm{~mm}$ of diameter and particle of $5 \mu \mathrm{m}$ (ThermoFisher Scientific). The temperature of column stove was kept at $40{ }^{\circ} \mathrm{C}$ and the wave length at $266 \mathrm{~nm}$ for detection by ultraviolet. The run rime was defined at $10 \mathrm{~min}$. Samples were prepared at the concentration of $1 \mathrm{mg} \cdot \mathrm{mL}^{-1}$ using methanol as solvent and posteriorly they were diluted for the concentration of $0.2 \mathrm{mg} \cdot \mathrm{mL}^{-1}$ using the mobile phase as diluent.

The analytical method was validated according to the guidelines of ICH Q2(R1) (ICH 2005). The parameters selectivity, limit of quantification, linearity, intra- and inter-day precision, accuracy, range and robustness were evaluated.

\section{RESULTS AND DISCUSSION}

\section{Characterization}

Figure 2 presents the diffraction patterns obtained by PXRD for Telzir ${ }^{\circledR}$, fosamprenavir calcium extracted from this formulation and for the drug heated at $100{ }^{\circ} \mathrm{C}$ during $5 \mathrm{~h}$ at vacuum. There is a perfect overlap between the Bragg peaks from the diffractograms of Telzir ${ }^{\circledR}$ and the extracted drug. This indicates that the drug recrystallization when submitted 


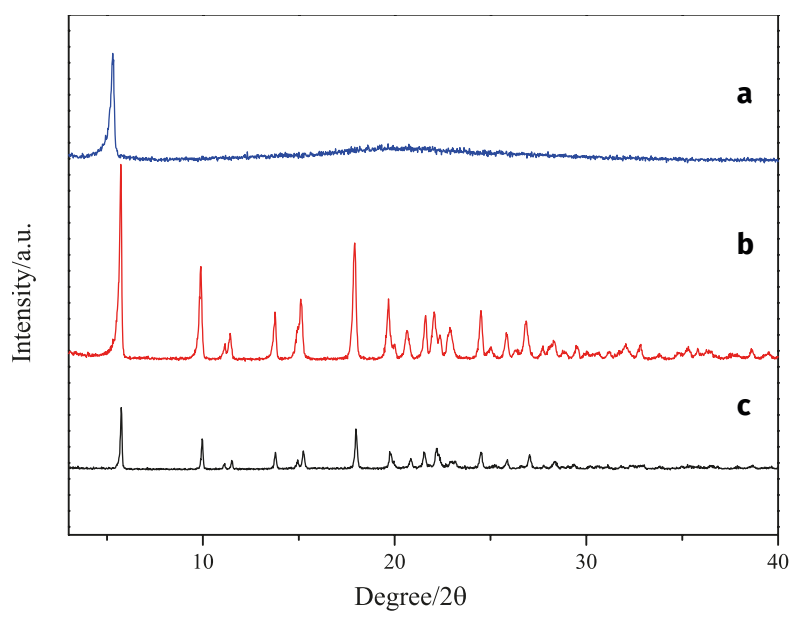

Figure 2. Diffractogram of the solid forms of fosamprenavir obtained by PXRD: $3^{\circ}-40^{\circ} 2 \theta, 40 \mathrm{KV}, 30$ $m A, C u K \alpha=1.54178$. (a) Amorphous form; (b) Form I; (c) Reference medicine.

to extraction did no alter its crystalline form, which correspond to those described as Form I (Armitage et al. 2003, Bhoge et al. 2012). This crystalline phase presented peaks at 5.74, 9.92, 11.44, 13.78, 15.14, 17.93, 19.70, 20.66, 21.62, 22.06, 22.90, 24.52 and $26.822^{\circ} \theta$. On the other hand there were no Bragg peaks in the diffractogram obtained for the heated fosamprenavir calcium, thus indicating the loss of long-range ordered arrangement of the drug molecules, thus characterizing an amorphous substance.

Figure 3 presents thermoanalytical curves obtained for the solid forms of fosamprenavir calcium used in the present study. For both forms the first event of mass loss occurs in the range between $30{ }^{\circ} \mathrm{C}$ and $147.5^{\circ} \mathrm{C}$. In this temperature range Form I presented a loss of $13.47 \%$ corresponding to the elimination of 5 molecules of water present in this solid form, which is described as a pentahydrate. This content of water was confirmed by titration analysis in Karl Fischer apparatus (Metrohm, São Paulo, Brazil). The amorphous form presented a loss of $4.6 \%$, indicating two molecules of water and a partial dehydration of Form I when submitted to the described conditions.
The second event of mass loss in both forms started at approximately $170{ }^{\circ} \mathrm{C}$ and it intensifies after $282{ }^{\circ} \mathrm{C}$, that indicates that the fosamprenavir calcium suffers thermal degradation before the fusion point described in the literature $\left(282^{\circ} \mathrm{C}-284^{\circ} \mathrm{C}\right)$ (Merck 2013), and therefore the drug does not present defined temperature neither enthalpy of fusion.

The first endothermic event of DSC curve observed for both forms corresponds to the first event of mass loss of TGA curves, what is equivalent to the elimination of water molecules present in the solid form of the drug. The thermal profiles present this first endothermic peak at $86.53{ }^{\circ} \mathrm{C}\left(T_{\text {onset }}=62.00^{\circ} \mathrm{C}\right.$ and enthalpy $\left.\Delta H=287.8 \mathrm{~J} . \mathrm{g}^{-1}\right)$ and $68.40{ }^{\circ} \mathrm{C}\left(T_{\text {onset }}\right.$ $=44.70{ }^{\circ} \mathrm{C}$ and $\left.\Delta H=77.62 \mathrm{~J} \cdot \mathrm{g}^{-1}\right)$ for Form I and amorphous form, respectively. The enthalpy of Form I is 3.7 times higher than amorphous due crystalline form have more molecules of water than amorphous. The other two endothermic events are verified at the DSC curve, which were equivalent for Form I and amorphous form and correspond to the drug degradation, as verified in the thermogravimetric analysis.

DSC curve of crystalline form did not show a fusion peak, indicating that crystalline structure collapses after dehydration process, thus reinforcing the need for such molecules to

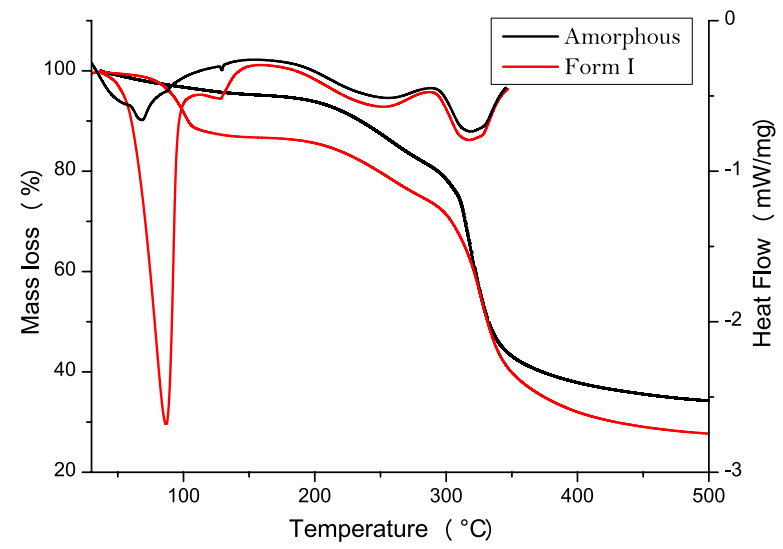

Figure 3. TGA and DSC curves of Fosamprenavir Form I (red) and amorphous (black). 
maintain the crystalline structure of the Form I. Such evidences are corroborated by the results obtained through PXRD and TGA.

Spectra of FTIR obtained for Form I and amorphous fosamprenavir at room temperature are presented on Figure 4. Despite the absorption bands were similar in both forms, a lower intensity and greater enlargement of bands corresponding to the amorphous form are verified, which is characteristic of substances with low or any crystallinity (Beraldi et al. 2014).

The main absorption bands characteristic of fosamprenavir are described as follows: region $\mathrm{A}, \mathrm{O}-\mathrm{H}$ stretches of water molecules and medium intensity $\mathrm{N}-\mathrm{H}$ stretches of amine and amide functions; 2975.6 and $2867.5 \mathrm{~cm}^{-1}$, aliphatic $\mathrm{C}-\mathrm{H}$ stretches of medium to high intensity; 1669.2 $\mathrm{cm}^{-1}$, high intensity $\mathrm{C}=\mathrm{O}$ stretches of the carbonyl

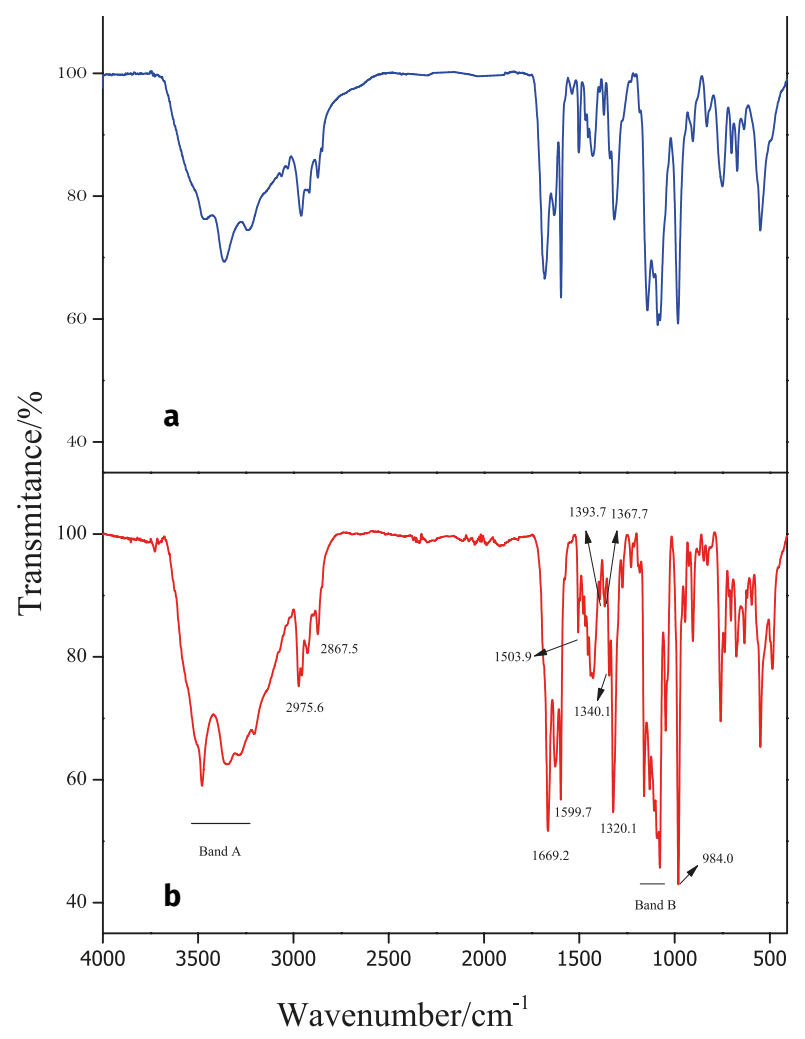

Figure 4. Infrared spectra obtained for solid forms of fosamprenavir (room temperature, interval from 4000 to $400 \mathrm{~cm}^{-1}, 32$ scans, resolution of $4 \mathrm{~cm}^{-1}$ ). (a) Amorphous form; (b) Form I. group; 1599.7 and $1503.9 \mathrm{~cm}^{-1}$, medium intensity $\mathrm{C}=\mathrm{C}$ stretches of the aromatic ring; $1393.7 \mathrm{~cm}^{-1}$, medium intensity asymmetrical folding of the isopropyl group; $1367.7 \mathrm{~cm}^{-1}$, medium intensity symmetrical folding of the isopropyl group; $1340.1 \mathrm{~cm}^{-1}, \mathrm{C}-\mathrm{N}$ stretch of medium to high intensity of the amine function; $1320.1 \mathrm{~cm}^{-1}$, high intensity asymmetrical stretch $\mathrm{S}=\mathrm{O}$; region $\mathrm{B}$, high intensity $\mathrm{C}-\mathrm{O}$ stretch of ester and ether functions; $984 \mathrm{~cm}^{-1}$, high intensity C-O stretch of phosphate ester (Pavia et al. 2013).

The morphology of crystalline Form I of fosamprenavir was determined by scanning electron microscopy. Images presented on Figure 5 were obtained with magnification of 4000 , 8000 and 13000 times and demonstrate that this form is composed by microcrystalline needles (RSC 2013). SEM analysis of amorphous material (Reddy et al. 2015) do not release any features and no particles can be distinguished, even at 5000x of magnification, of crystalline forms. This analysis is relevant for characterizing solid forms, once the morphology of drugs implies directly on its physical properties such as fluidity, compressibility, stability in suspension and dissolution.

\section{Studies of stability}

Studies of stability were conducted applying the Form I of fosamprenavir, the crystalline phase found in the commercial product Telzir ${ }^{\circledR}$. The chemical and polymorphic stability of the drug under controlled conditions of temperature and humidity were evaluated according to the guidelines of ICH (International Conference on Harmonization) (ICH 2003) and ANVISA (Anvisa 2012) for studies of accelerate stability of active pharmaceutical ingredients. Samples were initially evaluated by the techniques DSC, PXRD, FTIR and HPLC. Posteriorly they were incubated in stability chamber at $40^{\circ} \mathrm{C} \pm 2^{\circ} \mathrm{C}$ and relative 

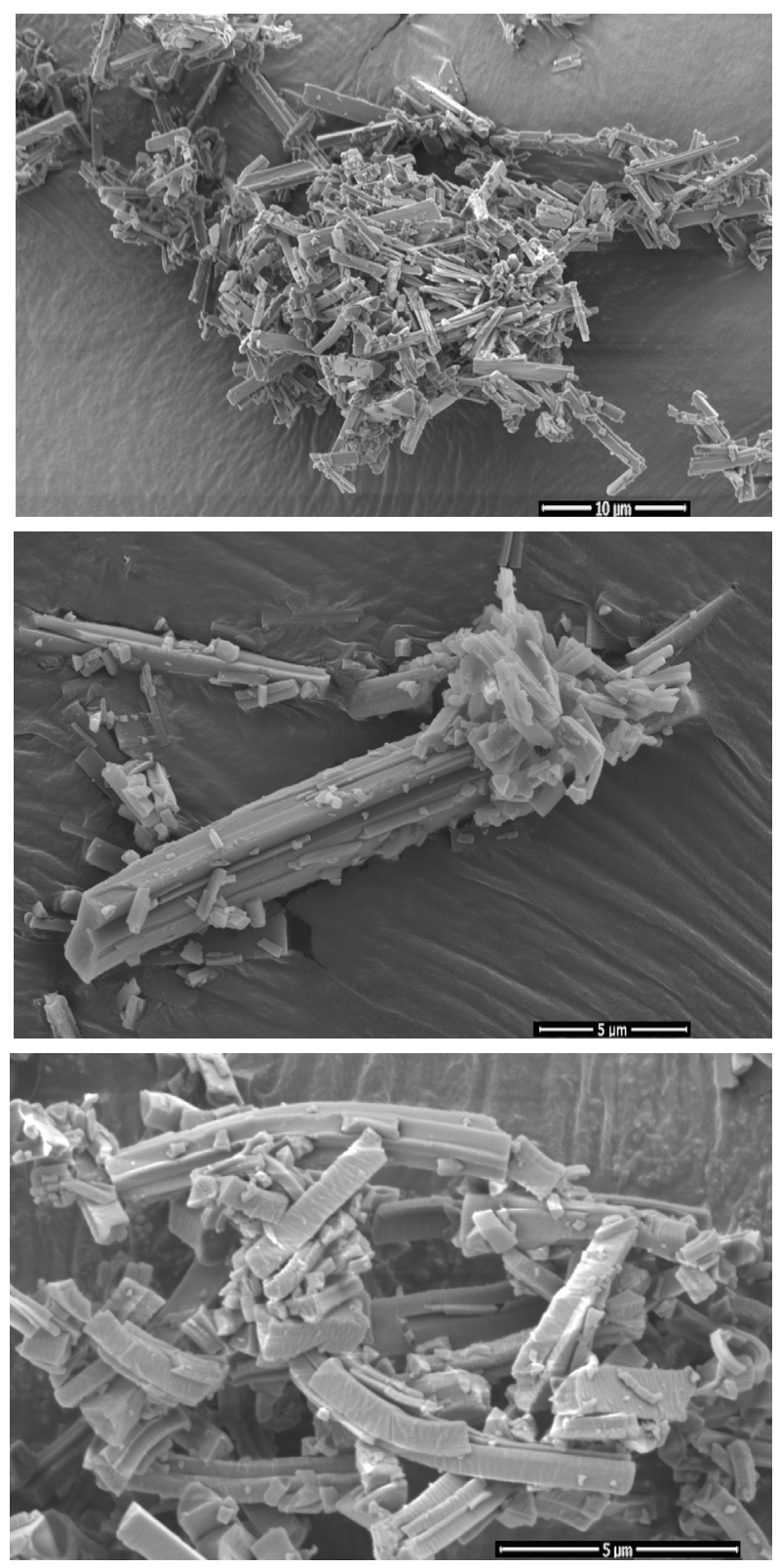

Figure 5. Electromicrographs of fosamprenavir Form I: magnification of (a) $4000 \mathrm{x}$, (b) $8000 \mathrm{x}$ and $13000 \mathrm{x}$.

humidity of $75 \% \pm 5 \%$ and analyzed again after three and six months of incubation.

DSC curves obtained for the fosamprenavir at time zero (sample not submitted to stressing conditions) and after three and six months of incubation are presented on Figure 6. Since the assays of characterization revealed that the drug did not present a well-defined endothermic peak of fusion in the DSC curve due all its observed thermal events are associate with mass loss (dehydration or molecule decomposition). The maintenance of Form I crystallinity was evaluated using enthalpy values of the endothermic peak corresponding to the drug dehydration, which are presented on Table 1. Significant differences were not verified among the values of enthalpy obtained throughout the stability study, what suggests that Form I remained stable in the evaluated storage conditions.

Diffraction patterns obtained for Form I of fosamprenavir during stability studies are presented on Figure 7. The diffractogram overlap allows verifying that Bragg peaks occurred at the same diffraction angles, thus indicating that there is no difference of crystalline structure among samples. Such results are in accordance with those observed in the thermal analysis by DSC and prove the polymorphic stability of Form I of fosamprenavir under the evaluated conditions of temperature and humidity.

Infrared spectroscopy was used as complementary technique in the study of chemical and polymorphic stability of Form I. Figure 8 presents a comparison among spectra obtained for samples in the time zero and after three and six months of incubation. The spectra were similar for all samples and alterations in the mode of vibrational frequency of functional groups present in the molecules of the drug were not observed, what highlights the polymorphic stability of Form I and corroborates results obtained by DSC and PXRD. Moreover, the appearance or disappearance of absorption bands was not observed in the spectra of samples of three and six months of incubation, thus suggesting that there was no chemical degradation of the drug while exposed to the evaluated storage conditions.

The confirmation of chemical stability of Form I of fosamprenavir was made through 
the chromatographic method indicative of stability for the drug dosing and quantification of degradation products. HPLC and the method validation proved its suitability for the desired purposes. Results obtained for the drug dosing are presented on Table 2. There were

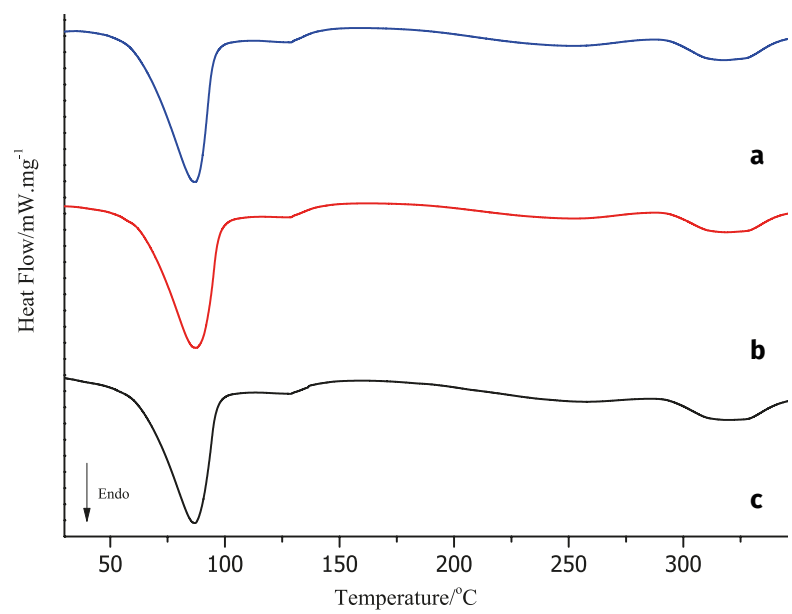

Figure 6. DSC curves obtained for Form I of fosamprenavir submitted to the stability study (temperature of $40^{\circ} \mathrm{C} \pm 2{ }^{\circ} \mathrm{C}$ and relative humidity of $75 \% \pm 5 \%$ ). (a) Sample at time zero (without stress); (b) three months of incubation; (c) six months of incubation. no significant differences among results and the formation of degradation products was not observed in any analyzed sample. Such results prove the chemical stability of Form I in accordance with those obtained by FTIR.

Table I. Enthalpy values obtained for the first endothermic event of Form I submitted to the stability study

\begin{tabular}{|c|c|}
\hline Time & $\mathbf{\Delta H}\left(\mathbf{J . g}^{-1}\right)$ \\
\hline Initial & 287.8 \\
\hline 3 months & 287.9 \\
\hline 6 months & 286.9 \\
\hline
\end{tabular}

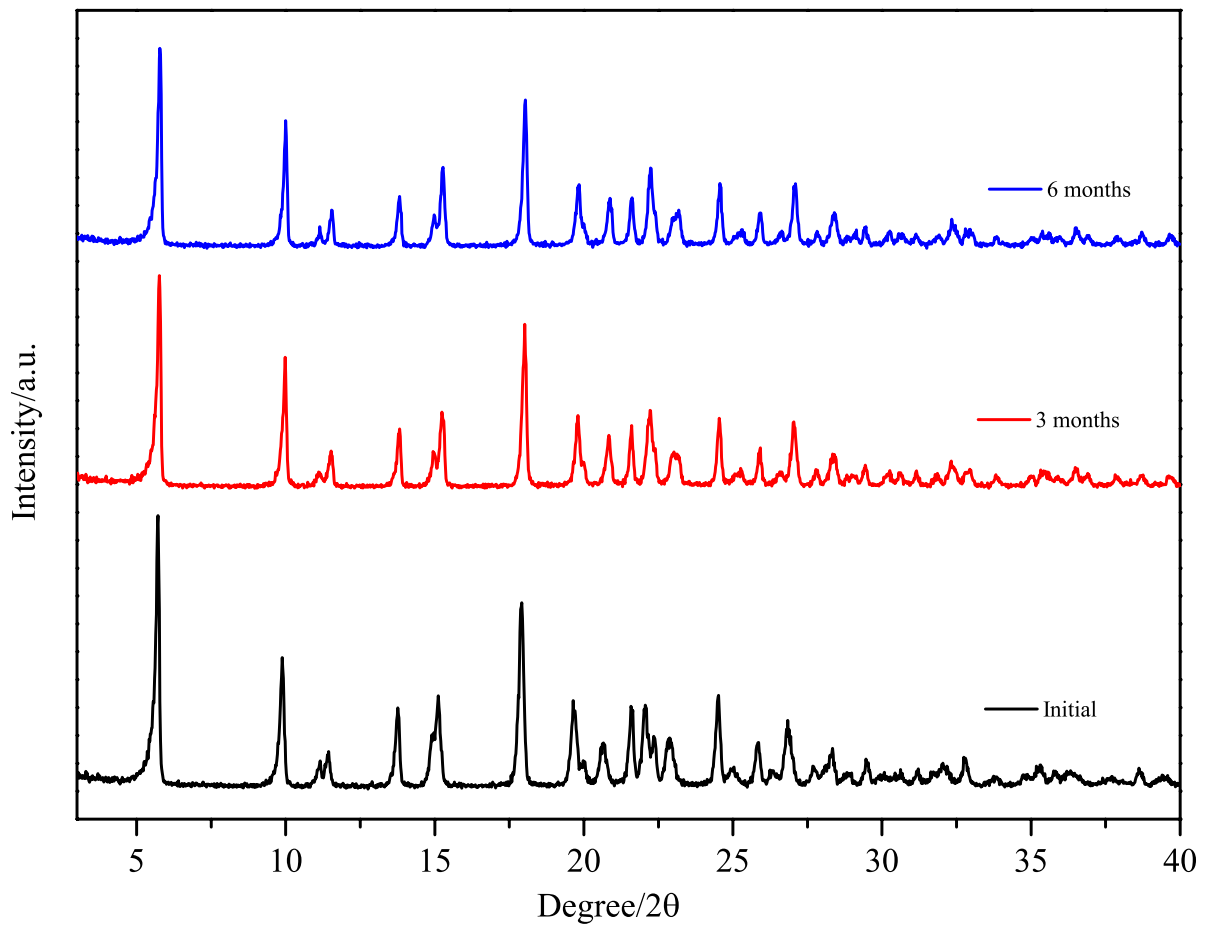

Figure 7. Diffractogram obtained for Form I of fosamprenavir submitted to stability study (temperature of $40^{\circ} \mathrm{C} \pm 2{ }^{\circ} \mathrm{C}$ and relative humidity of $75 \% \pm 5 \%$ ). Initial sample (without stress), three months of incubation, six months of incubation. 


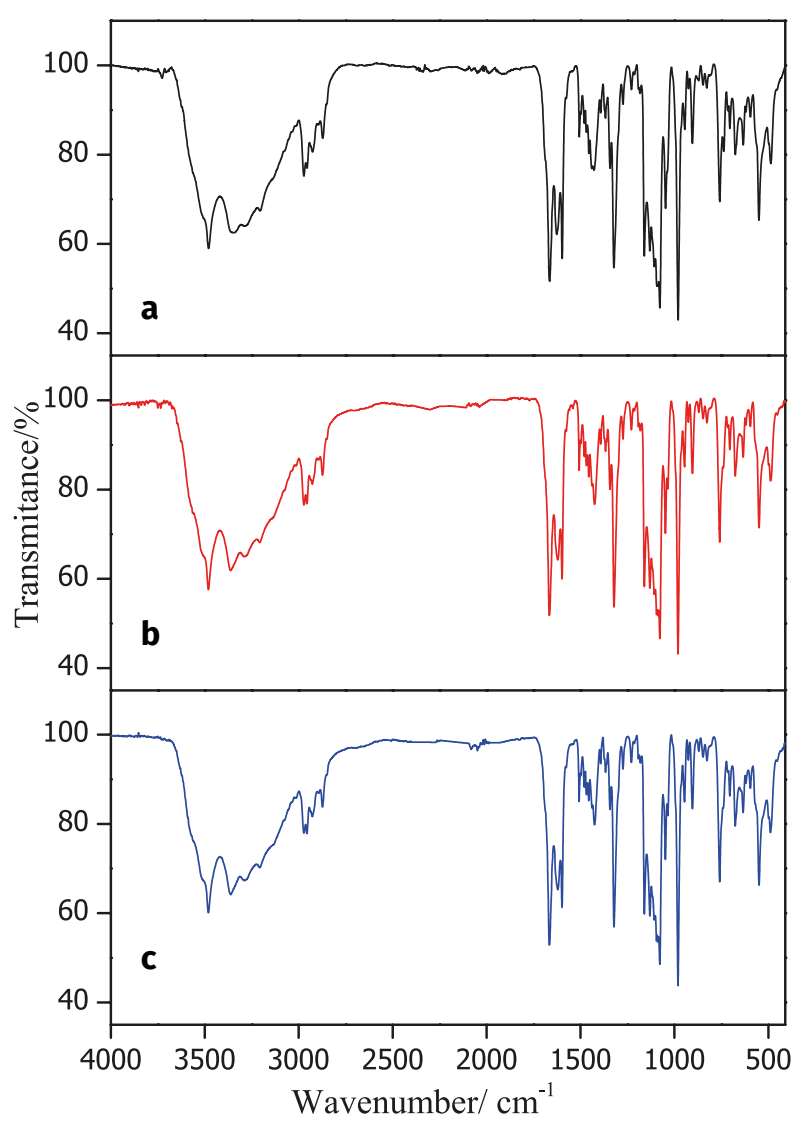

Figure 8. Infrared spectra obtained for Form I of fosamprenavir submitted to stability study (temperature of $40^{\circ} \mathrm{C} \pm 2{ }^{\circ} \mathrm{C}$ and relative humidity of $75 \% \pm 5 \%$ ). (a) Initial sample (without stress); (b) three months of incubation; (c) six months of incubation.

Table II. Fosamprenavir dosing (mean $\pm \mathrm{Cl}, n=3$ and $\alpha=0.05$ ) in samples of Form I submitted to stability study. Initial sample (without stress), three months of incubation and six months of incubation.

\begin{tabular}{|c|c|}
\hline Time & Assay (\%) \\
\hline Initial & $100.8 \pm 2.5$ \\
\hline 3 months & $101.2 \pm 3.3$ \\
\hline 6 months & $101.2 \pm 3.6$ \\
\hline
\end{tabular}

\section{CONCLUSIONS}

The fosamprenavir calcium was efficiently isolated from the medicine Telzir ${ }^{\circledR}$. Analyses by PXRD demonstrated that the crystalline phase of the drug obtained by extraction corresponds to the phase found in the commercial product, called Form I. The posterior heating of Form I at $100{ }^{\circ} \mathrm{C}$ generated an amorphous solid form of the fosamprenavir.

Forms obtained for the drug were characterized by the techniques TGA, DSC, PXRD, FTIR and SEM. The thermo-analytical methods characterize Form I of fosamprenavir as a pentahydrate. Furthermore, these methods associated to the techniques PXRD and FTIR allowed concluding that the presence of water in Form I is fundamental for the maintenance of the crystalline structure. Therefore, the use of thermal techniques is crucial for monitoring the crystallinity of this active principle and the warranty of its pharmaceutical efficiency.

In studies of stability Form I of fosamprenavir presented suitable characteristics to be used in pharmaceutical preparations. Alterations in the chemical or polymorphic stability were not detected when submitted to conditions of temperature and humidity recommended for studies of accelerate stability of active pharmaceutical ingredients. However conditions of transport and storage of fosamprenavir must be carefully controlled since the exposition to elevate temperatures may cause the loss of crystallinity, thus generating amorphous forms of the drug.

Results obtained in the present study employing the techniques of DSC, TGA, PXRD, FTIR and HPLC presented a good correlation. Thermo-analytical methods associated to spectroscopic and chromatographic techniques seem to be an important tool in studies of characterization and stability of drugs. Thermal analyses provide simple and fast results relevant for the providence of secure, efficient and high quality pharmaceutical products. 


\section{Acknowledgments}

Authors thank Fundação de Amparo à Pesquisa do Estado de Minas Gerais (FAPEMIG), Coordenação de Aperfeiçoamento de Pessoal de Nivel Superior (CAPES) and Laboratory of Crystallography UNIFAL/MG.

\section{REFERENCES}

ANVISA - BRAZIL'S NATIONAL HEALTH SURVEILLANCE AGENCY. 2012. Resolution RE n 45. http://www.anvisa.gov.br. Accessed 07 Feb 2020.

ARMITAGE IG, SEARLE AD \& SINGH H. 2003. Calcium (3S) tetrahydro-3-furanyl (1S,2R)-3-[[(4-aminophenyl) sulfonyl](isobutyl)amino]-1-benzyl-2-(phosphonooxy) propylcarbamate. US patent. 6514953 B1.

BERALDI C, TINTI A, OTTANI S \& GAMBERINI MC. 2014. Characterization of polymorphic ampicillin forms. J Pharm Biomed Anal 100: 329-340.

BHOGE SM, KSHIRSAGAR P, RICHHARIYA S \& SINGH K. 2012. Crystalline form of fosamprenavir calcium. US patent. 0208787 A1.

CHADHA R \& BHANDARI S. 2014. Drug-excipient compatibility screening - Role of thermoanalytical and spectroscopic techniques. J Pharm Biom Anal 87: 82-97.

CORRÊA JCR, PERISSINATO AG, SERRA CHR, TREVISAN MG \& SALGADO HRN. 2016. Polymorphic stability of duranavir and its formulation. J Therm Anal Calorim 123: 2185-2190.

DANIEL JSP, VERONEZ IP, RODRIGUES LL, TREVISAN MG \& GARCIA JS. 2013. Risperidone - Solid-state characterization and pharmaceutical compatibility using thermal and nonthermal techniques. Therm Acta 568: 148-155.

ICH - INTERNATIONAL CONFERENCE ON HARMONIZATION. 2003. Guidance for industry Q1A(R2): Stability testing of new drug substances and products. http://www.ich.org/ home.html. Accessed 07 Feb 2020.

ICH - INTERNATIONAL CONFERENCE ON HARMONIZATION. 2005. Guidance for industry ICH Q2(R1): Validation of analytical procedures: Text and Methodology. http://www.ich.org/ home.html. Accessed 07 Feb 2020.

JETTI RR, BHAGAVATULA N \& GORANTLA AR. 2013. Crystalline fosamprenavir calcium and process for the preparation thereof. US patent. 0317236 A1.

JÚLIO TA, GARCIA JS, BONFILIO R, ARAÚJO MB \& TREVISAN MG. 2015. Solid-state stability and solubility determination of crystalline forms of Moxifloxcin Hydrochloride. Int J Pharm Pharmac Sci 7: 173-177.
LEKSIC E, SEPAC D \& PAVLICIC D. 2011. Solid state forms of fosamprenavir calcium salt and processes for preparation thereof. US patent. 0165202 A1.

PÁVIA DL, KRIZ GS, LAMPMAN GM \& VYVYAN JR. 2013. Introduction to Spectroscopy. $4^{\text {th }}$ ed., Cengage Learning.

REDDY BP, REDDY KR, REDDY DM, REDDY SC \& KRISHNA BV. 2015. Novel polymorphs of fosamprenavir calcium. US patent. 0025040 A1.

ROSSI RC, DIAS CL, BAJERSKI L, BERGOLD AN \& FROEHLICH PE. 2011. Development and validation of discriminating method of dissolution for fosamprenavir tablets based on in vivo data. J Pharm Biomed Anal 54: 439-444.

RSC - THE ROYAL SOCIETY OF CHEMISTRY. 2013. The Merck Index. $15^{\text {th }}$ ed., Publishing.

SANTOS OMM, FREITAS JTJ, CAZEDEY ECL, ARAÚJO MB \& DORIGUETTO AC. 2016. Structure, solubility and stability of Orbifloxacin crystal forms: hemihydrate versus anhydrate. Molecules 21: 328.

SHETE G, PURI V, KUMAR L \& BANSAL AK. 2010. Solid state characterization of commercial and amorphous Atorvastatin calcium samples. AAPS PharmSciTech 11: 598-609.

SINGH S, HANDA T, NARAYANAM M, SAHU A, JUNWAL M \& SHAH P. 2012. A critical review on the use of modern sophisticated hyphenated tools in the characterization of impurities and degradation products. J Pharm Biom Anal 69: 148-173.

WHO - WORLD HEALTH ORGANIZATION. 2002. The importance of pharmacovigilance, http://apps.who.int/iris/ bitstream/10665/42493/1/a75646.pdf. Accessed 07 Feb 2020.

\section{How to cite}

CORDEIRO CF, BETTIO I \& TREVISAN MG. 2020. Studies on the characterization and polymorphic stability of Fosamprenavir. An Acad Bras Cienc 92: e20181021. DOI 10.1590/0001-3765202020181021.

Manuscript received on October 4, 2018; accepted

for publication on October 24, 2018

\section{CLEYDSON F. CORDEIRO}

https://orcid.org/0000-0002-7564-1756

\section{INGRID BETTIO}

https://orcid.org/0000-0003-0566-6090

\section{MARCELLO G. TREVISAN}

https://orcid.org/0000-0002-5960-9369 
LACFar, Institute of Chemistry, Federal University of

Alfenas, Av. Jovino Fernandes Sales, 2600, Bairro

Santa Clara, 37130-000 Alfenas, MG, Brazil

Correspondence to: Marcello Garcia Trevisan

E-mail:mgtrevisan@gmail.com

\section{Author contributions}

Cordeiro, CF wrote the content of the paper, conducted the experiments involving HPLC and PXRD and processed the results. Bettio I contributed in Thermal Analysis and FTIR Spectroscopy. Trevisan, MG revised the format of the paper and figures and contributed for interpretation of the data. All authors provided critical discussion about results showed in the final version of manuscript.

\section{(cc) BY}

